Article

\title{
Functional Analysis of the Isopentenyl Diphosphate Isomerase of Salvia miltiorrhiza via Color Complementation and RNA Interference
}

\author{
Xianan Zhang ${ }^{1, \dagger}$, Hongyu Guan ${ }^{1, \dagger}$, Zhubo Dai ${ }^{2}$, Juan Guo ${ }^{3}$, Ye Shen ${ }^{3}$, Guanghong Cui ${ }^{3}$, \\ Wei Gao ${ }^{1}$ and Luqi Huang ${ }^{3, *}$ \\ Received: 28 September 2015 ; Accepted: 3 November 2015 ; Published: 10 November 2015 \\ Academic Editor: Marcello Iriti \\ 1 School of Traditional Chinese Medicine, Capital Medical University, Beijing 100069, China; \\ zhxn0609@163.com (X.Z.); 13716402100@139.com (H.G.); weigao@ccmu.edu.cn (W.G.) \\ 2 Key Laboratory of Systems Microbial Biotechnology, Tianjin Institute of Industrial Biotechnology, \\ Chinese Academy of Sciences, Tianjin 300308, China; dai_zb@tib.cas.cn \\ 3 Institute of Chinese Materia Medica, Chinese Academy of Chinese Medical Sciences, Beijing 100700, \\ China; guojuanzy@163.com (J.G.); shenyezy@sina.com (Y.S.); guanghongcui@126.com (G.C.) \\ * Correspondence: huangluqi68@163.com; Tel.: +86-10-6401-4411; Fax: +86-10-6401-3996 \\ $\dagger$ These authors contributed equally.
}

\begin{abstract}
Isopentenyl diphosphate isomerase (IPI) catalyzes the isomerization between the common terpene precursor substances isopentenyl diphosphate (IPP) and dimethylallyl diphosphate (DMAPP) during the terpenoid biosynthesis process. In this study, tissue expression analysis revealed that the expression level of the Salvia miltiorrhiza IPI1 gene (SmIPI1) was higher in the leaves than in the roots and stems. Furthermore, color complementation and RNA interference methods were used to verify the function of the SmIPI1 gene from two aspects. A recombinant SmIPI1 plasmid was successfully constructed and transferred into engineered E. coli for validating the function of SmIPI1 through the color difference in comparison to the control group; the observed color difference indicated that SmIPI1 served in promoting the accumulation of lycopene. Transformant hairy root lines with RNA interference of SmIPI1 were successfully constructed mediated by Agrobacterium rhizogenes ACCC 10060. RNA interference hairy roots had a severe phenotype characterized by withering, deformity or even death. The mRNA expression level of SmIPI1 in the RSi3 root line was only $8.4 \%$ of that of the wild type. Furthermore the tanshinone content was too low to be detected in the RNA interference lines. These results suggest that SmIPI1 plays a critical role in terpenoid metabolic pathways. Addition of an exogenous SmIPI1 gene promoted metabolic flow toward the biosynthesis of carotenoids in E. coli, and SmIPI1 interference in S. miltiorrhiza hairy roots may cause interruption of the 2-C-methyl-D-erythritol-4-phosphate metabolic pathway.
\end{abstract}

Keywords: isopentenyl diphosphate isomerase; color complementation; RNA interference; Salvia miltiorrhiza; functional identification

\section{Introduction}

Salvia miltiorrhiza Bunge is a traditional Chinese medicine that is frequently used for curing cardiovascular diseases, and its preparations are currently in phase II clinical trials [1]. S. miltiorrhiza has documented antibacterial, antiphlogosis, thrombosis, myocardial ischemia, hepatic, and antioxidative properties [2], and the tanshinones (such as tanshinone IIA, dihydrotanshinone I, cryptotanshinone, and tanshinone I) are regarded as essential components for these activities. 
Moreover, S. miltiorrhiza has been prominently mentioned in discussions of modern approaches for studying traditional Chinese medicine [3].

Tanshinone is a diterpene compound synthesized from two C5 precursors, isopentenyl diphosphate (IPP) or its isomer dimethylallyl diphosphate (DMAPP), which is derived from the mevalonic acid (MVA) pathway in the cytoplasm and the 2-C-methyl-D-erythritol-4-phosphate (MEP) pathway in plasmids. In plants, IPP and DMAPP are catalyzed by several terpene synthases and cytochrome P450 enzymes in the downstream pathway to form more than 30,000 kinds of known terpenoids. Isopentenyl diphosphate isomerase (IPI) plays a major role in catalyzing the reversible conversions between the two terpenoid precursors [4] (Figure 1).

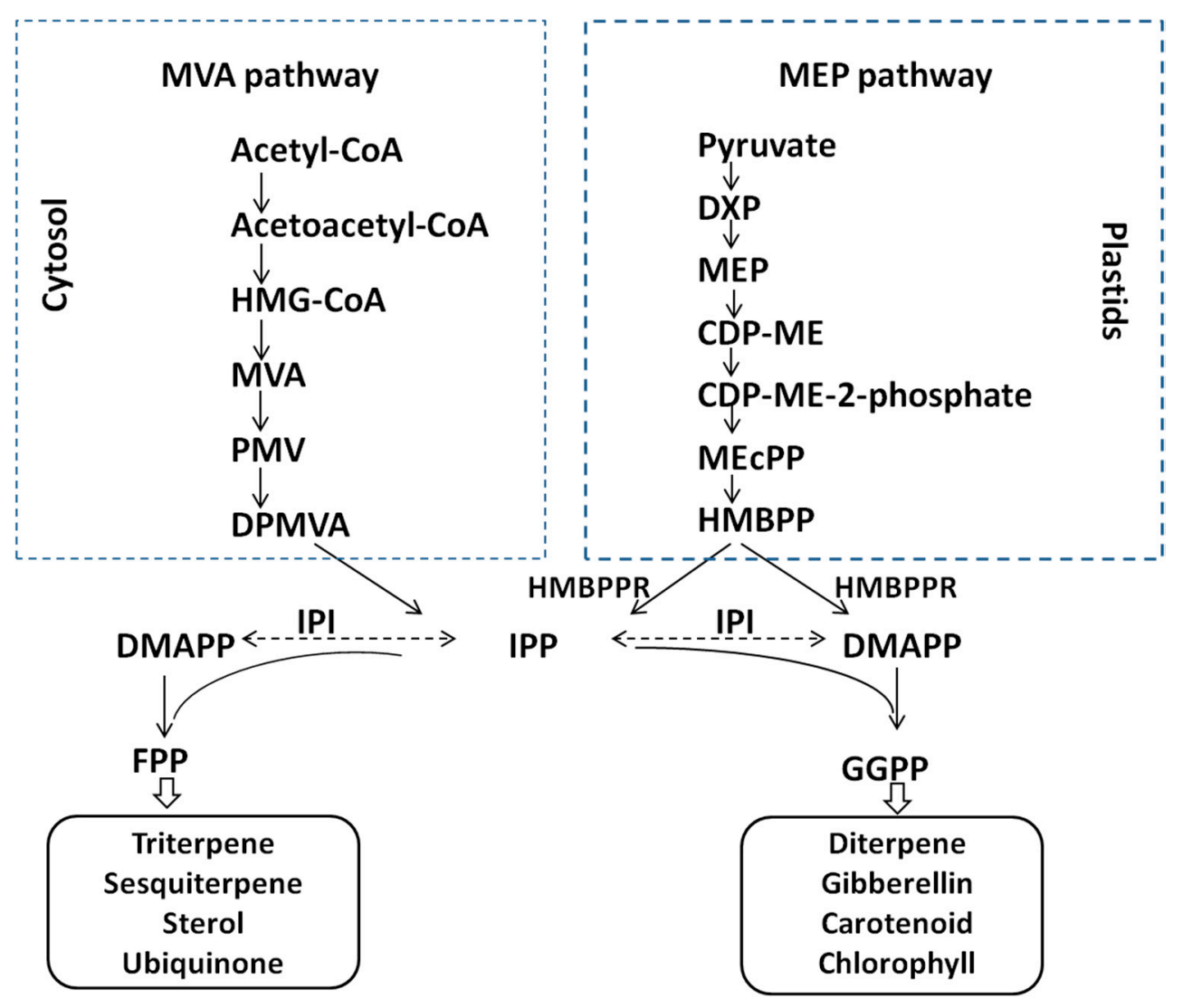

Figure 1. IPP and DMAPP synthesis pathway in plants. DMAPP is converted from IPP by IPI in the MVA pathway, whereas the simultaneous production of IPP and DMAPP from HMBPP occurs in the MEP pathway. HMG-CoA, 3-hydroxy-3-methylglutaryl CoA; MVA, mevalonic acid; IPP, isopentenyl diphosphate; DMAPP, dimethylallyl diphosphate; FPP, farnesyl diphosphate; GAP, glyceraldehyde 3-phosphate; DXP, 1-deoxy-d-xylulose-5-phosphate; MEP, 2-C-methyl-D-erythritol 4-phosphate; HMBPP, 1-hydroxy-2-methyl-2-(E)-butenyl 4-diphosphate; HMBPPR, 1-hydroxy-2-methyl-2-(E)butenyl-4-phosphate reductase; GGPP, geranylgeranyl diphosphate.

IPI is generally categorized into two distinct types: type I and type II. Type I IPI is utilized by most eukaryotes, some bacteria, and certain halophilic archaea, whereas type II IPI has been reported to be produced by bacteria such as Streptomyces [5], Bacillus [6], Cyanobacteria [7] and others. Most plants have two type I IPI isozymes with distinct subcelluar localizations [8]. At present, IPI cDNA has been obtained from many species [9-11], although their function is not necessarily the same.

According to previous reports, overexpression of external IPI cDNA in prokaryotic cells could lead to a dramatic increase in the content of carotenoids [12-15]. Silencing of the IPI gene in tobacco caused deficiency of photosynthetic pigments, and the reduction of IPI activity affected the synthesis 
of isoprene substances in tobacco plastids; however, few evident impacts have been observed with respect to the cytoplasmic MVA pathway [16].

In early studies, we selected multiple candidate genes involved in tanshinone biosynthesis in the hairy roots of $S$. miltiorrhiza, as revealed by a cDNA microarray [17] using 4400 expressed sequence tags, including SmIPI1. In order to confirm whether this gene is associated with the synthesis of tanshinones in S. miltiorrhiza, we conducted the following experiments: firstly, the SmIPI1 gene was transferred into lycopene-engineered Escherichia coli to determine whether expression of the gene would influence metabolic flow and promote the yield of lycopene. Secondly, RNA interference (RNAi) SmIPI1 hairy roots lines were established to evaluate the effect of SmIPI1 on tanshinone accumulation. These studies have the potential to increase our knowledge of the metabolism of terpenes formed through the MEP pathway.

\section{Results}

\subsection{Sequence Analysis of SmIPI cDNA}

The open reading frame of SmIPI1 cDNA was 918 bp, encoding 305 amino acid (aa) residues, with a relative molecular weight of $34.1 \mathrm{kDa}$ and theoretical isoelectric point of 6.13 . The amino acid sequence alignments showed that SmIPI1 has high homology with IPIs of other plant species. SmIPI1 contained a conservative cysteine motif TNTCCSHPL (155-163 aa) and a conservative WGEHELDY motif (217-224 aa) (Figure 2), which belong to the Type I IPIs. The BLAST comparison results indicated that the identity between SmIPI1 and SmIPI2 was $83 \%$, whereas it was $92 \%$ and $91 \%$ with Nicotiana tabacum IPI2 and IPI1, respectively. Relatively higher consistency was also seen with the IPI of Radix bupleuri (ACV74320) and Rhizoma picrorhizae (AB14800), which was 89\% and $90 \%$, respectively. Phylogenetic tree analysis showed that the IPIs of plants were clustered in a bigger branch, SmIPI1 is most closely genetically related with dicotyledon plants (Arabidopsis thaliana, Nicotiana tabacum etc.) (Figure 3).
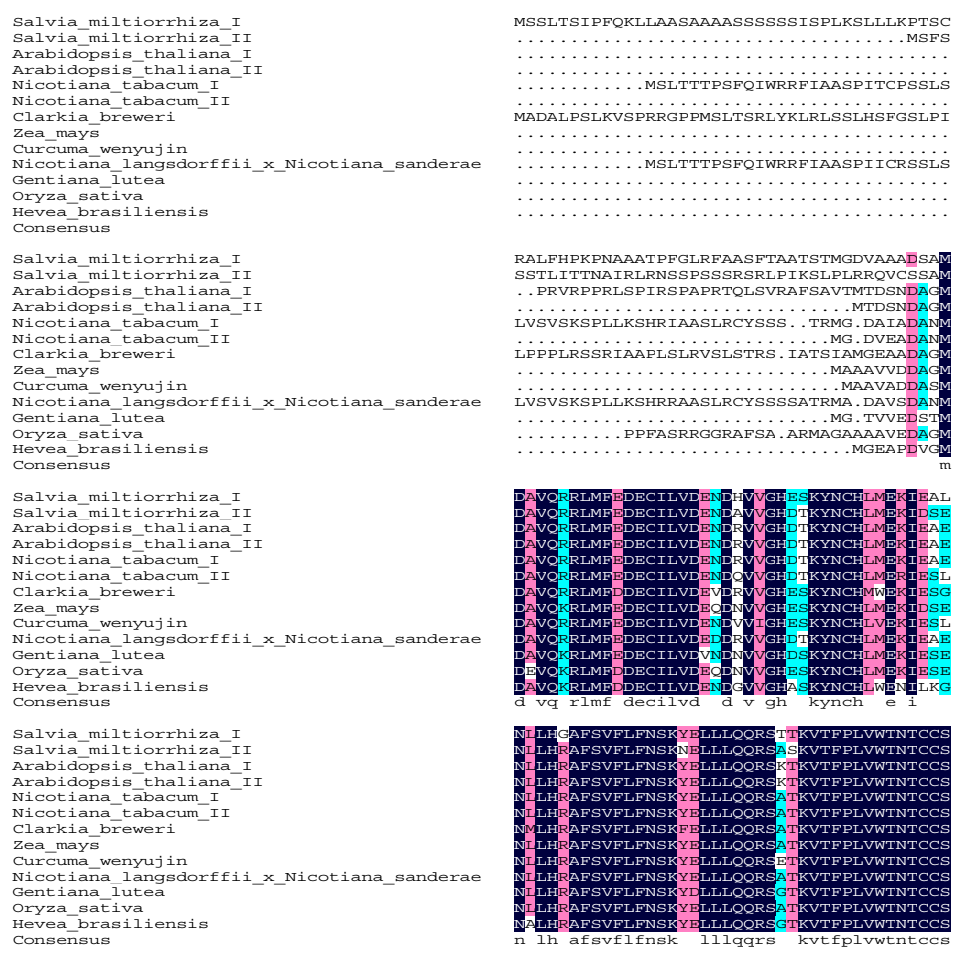

\begin{tabular}{r}
40 \\
4 \\
0 \\
0 \\
0 \\
28 \\
0 \\
40 \\
0 \\
0 \\
08 \\
28 \\
0 \\
0 \\
0 \\
\\
80 \\
80 \\
44 \\
38 \\
9 \\
65 \\
10 \\
79 \\
11 \\
10 \\
67 \\
10 \\
29 \\
9 \\
\\
120 \\
84 \\
78 \\
49 \\
105 \\
50 \\
119 \\
51 \\
50 \\
107 \\
50 \\
69 \\
49 \\
\\
\hline \\
160 \\
124 \\
118 \\
89 \\
145 \\
90 \\
159 \\
91 \\
90 \\
147 \\
90 \\
109 \\
89
\end{tabular}

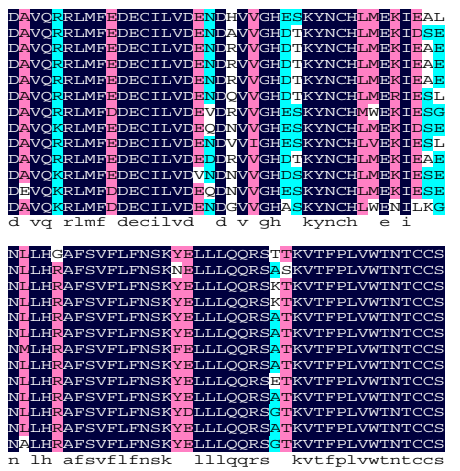

Figure 2. Cont. 

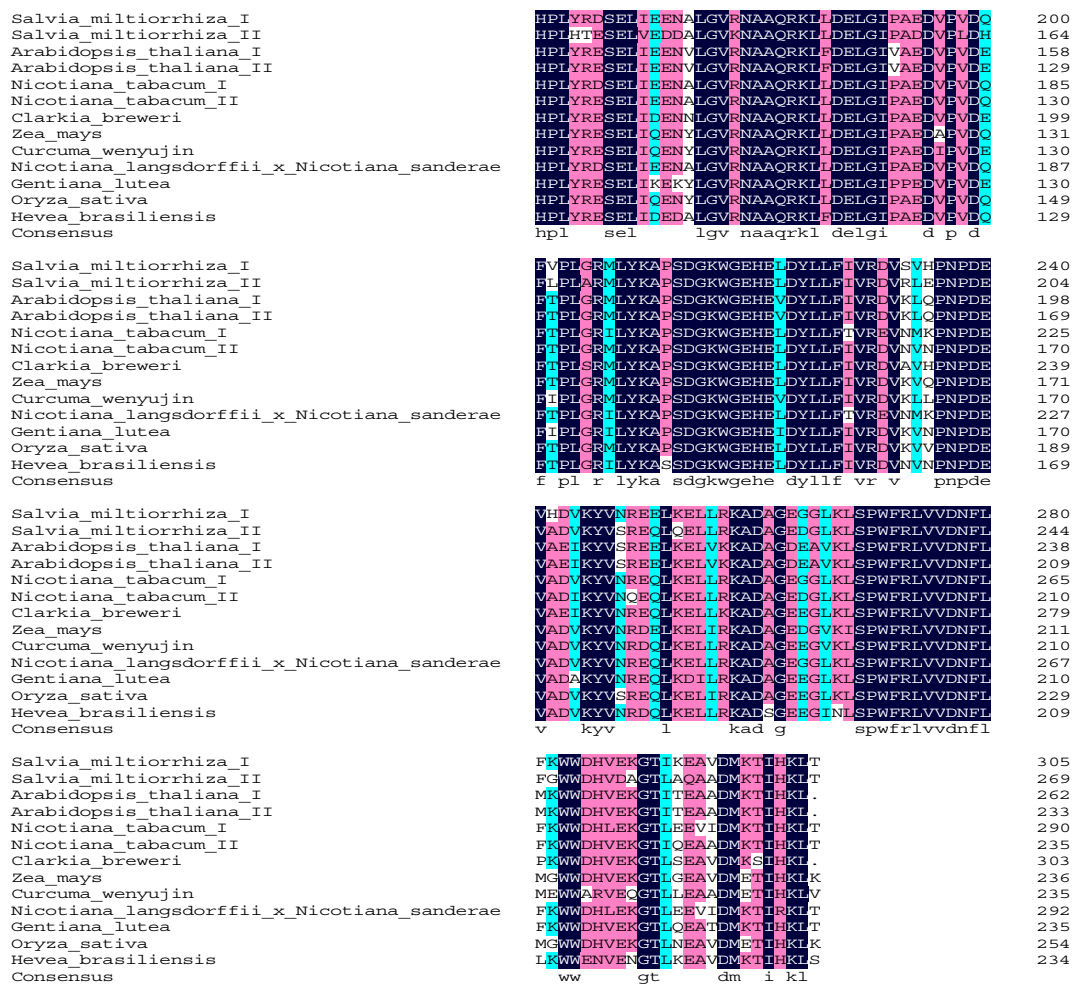

240
204
198
169
225
170
239
171
170
227
170
189
169

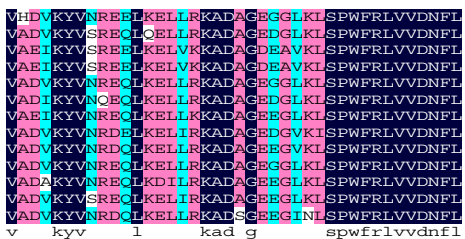

280
244
238
209
265
210
279
211
210
267
210
229
209

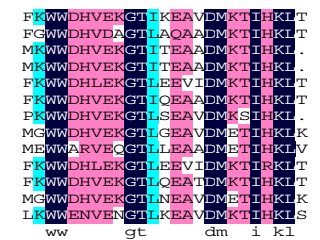

305
269
262
233
290
235
303
236
235
292
235
254
234

Figure 2. The amino acid sequence alignment of SmIPI1 with IPIs in other plants species. The amino acid accession numbers are as follows: Arabidopsis thaliana I AAB67741.1, Arabidopsis thaliana II AAL57687.1, Salvia miltiorrhiza I ABV08818.1, Salvia miltiorrhiza II JN831106.1, Nicotiana tabacum I BAB40973.1, Nicotiana tabacum II BAB40974.1, Clarkia breweri AAB67743.1, Zea mays AAQ14869.1, Curcuma wenyujin ADE05305.1, Nicotiana langsdorffii $\times$ Nicotiana sanderae ABB29847.1, Gentiana lutea BAE92733.1, Oryza sativa AAF29978, Hevea brasiliensis AAD41766.1.

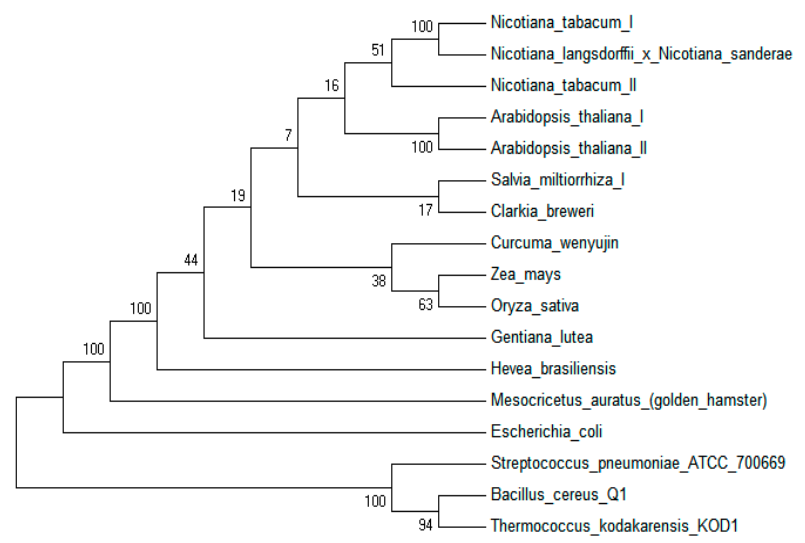

Figure 3. The phylogenetic tree was built based on the partial IPI amino acid sequences of different species using the neighbor-joining method. The amino acid sequence accession numbers are as follows: Arabidopsis thaliana I AAB67741.1, Arabidopsis thaliana II AAL57687.1, Salvia miltiorrhiza I ABV08818. 1, Nicotiana tabacum I BAB40973.1, Nicotiana tabacum II BAB40974.1, Clarkia breweri AAB67743.1, Zea mays AAQ14869.1, Curcuma wenyujin ADE05305.1, Nicotiana langsdorffii $\times$ Nicotiana sanderae ABB29847.1, Gentiana lutea BAE92733.1, Oryza sativa AAF29978, Hevea brasiliensis AAD41766.1, Escherichia coli AAD26812.1, Streptococcus pneumoniae ATCC 700669 B8ZLF5.1, Bacillus cereus Q1 B9IVM2.1, Mesocricetus auratus (golden hamster) O35586.1, Thermococcus kodakarensis KOD1 Q76CZ1.1. 


\section{2. mRNA Expression Analysis of SmIPI1 in Different Organs}

The SmIPI1 gene was expressed constitutively in all tissues examined with distinct levels. The highest level of SmIPI1 mRNA was observed in leaves, followed by roots and stems of S. miltiorrhiza (Figure 4A). Several experiments have shown that the addition of various elicitors could increase the yield of plant secondary metabolites to a certain degree, thus, the use of these elicitors are considered to be one of the most effective ways to improve the production of secondary metabolites of medicinal plants. Previous researches have indicated that the $\mathrm{Ag}^{+}$elicitor could promote the accumulation of diterpenoids of S. miltiorrhiza [18]. In this study, the relative expression level of SmIPI1 reached a peak at $24 \mathrm{~h}$ after addition of the $\mathrm{Ag}^{+}$elicitor and then declined from 36 to $120 \mathrm{~h}$ (Figure 4B).
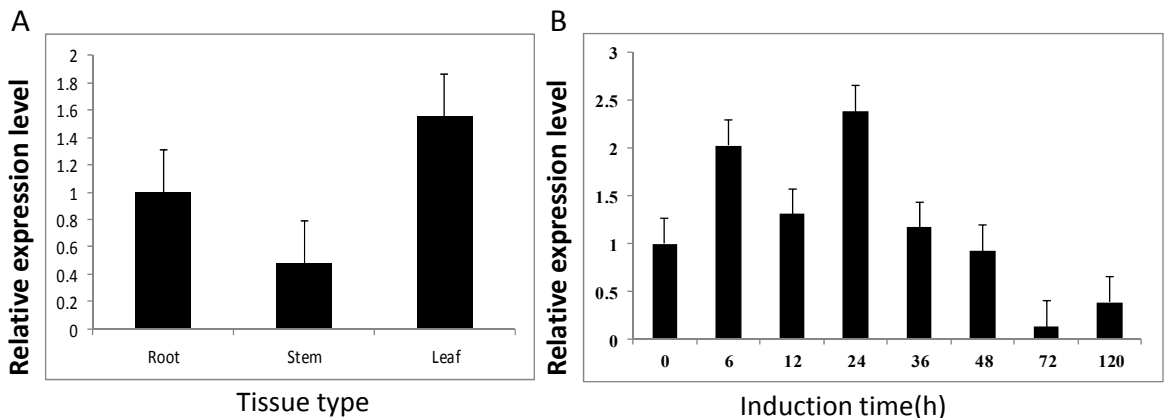

Figure 4. (A) Expression pattern of SmIPI1 in different S. miltiorrhiza tissues; (B) Expression profile of SmIPI1 after treatment with $30 \mathrm{mM} \mathrm{Ag}^{+}$over $120 \mathrm{~h}$. The vertical bars show the SD values $(n=3)$.

\subsection{Color Complementation Analysis of the SmIPI1 Gene}

The plasmid pAC-LYC, which was transferred into XL1-blue cells, acts to produce $\beta$-carotene [19]. In the double-resistant medium, E. coli strains harboring pTrc and the pAC-LYC plasmid could grow well, and the bacterial plaques appeared pink. pTrc-SmIPI1 along with pAC-LYC presented a deeper red color, which likely reflects its ability to accumulate more lycopene by the action of SmIPI1. On the other hand, the other three control strains could not grow in this double-resistant medium (Figure 5A). Thus, we can infer that SmIPI1 can promote the accumulation of lycopene in E. coli, and is a key enzyme in the carotenoid biosynthesis pathway.
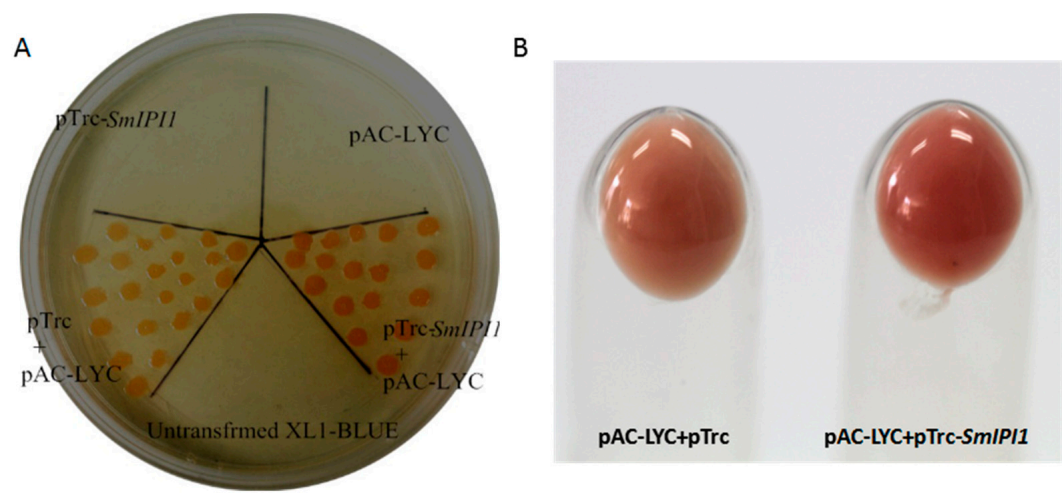

Figure 5. Color complementation and enhanced lycopene accumulation due to expression of SmIPI1 in engineered E. coli for lycopene biosynthesis. (A) The plate was divided into five sections, which were respectively inoculated with bacteria containing the corresponding plasmids as indicated on the plate; (B) The carroty bacterial clones including pTrc-SmIPI1 and pAC-LYC were darker because of lycopene accumulation. 


\subsection{Confirmation of Transgenic Plants by Fluorescent Microscopy and PCR Analysis}

Binary plasmids containing the target gene under the cauliflower mosaic virus $35 \mathrm{~S}$ promoter were transferred into S. miltiorrhiza via A. rhizogenes (ACCC 10060). Hairy roots emerged at the edge of the leaf disc after infection with $A$. rhizogenes harboring the binary vector pK7GWIWG2D-ipi (RSi). Hairy roots that were brown, thin and shorter were discarded, and only those with good growth capabilities were maintained for further characterization. Hairy roots that were successfully inserted with a target fragment emit a stable and strong green fluorescence signal $(520 \mathrm{~nm})$ under the inverted fluorescence microscope with the excitation of blue light $(450-490 \mathrm{~nm}$ ) (Figure 6A). Hairy roots from 1 month old transgenic cultures were used for DNA extraction and PCR-screening. An expected $733 \mathrm{bp}$ fragment of insert sequence (p35S-ipi) was amplified in the positive lines in our RNAi lines. WT hairy roots represent negative control and have no insertions and, therefore, no application of the fragment has been observed. The rolC gene of $540 \mathrm{bp}$ was amplified (Figure 6B).

A

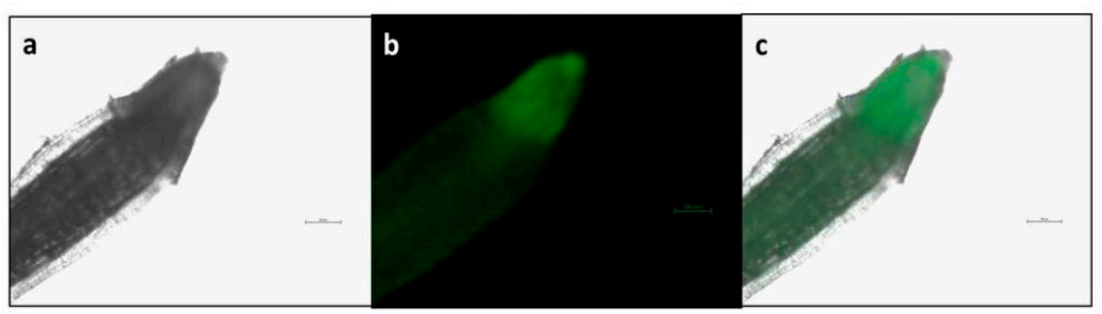

B

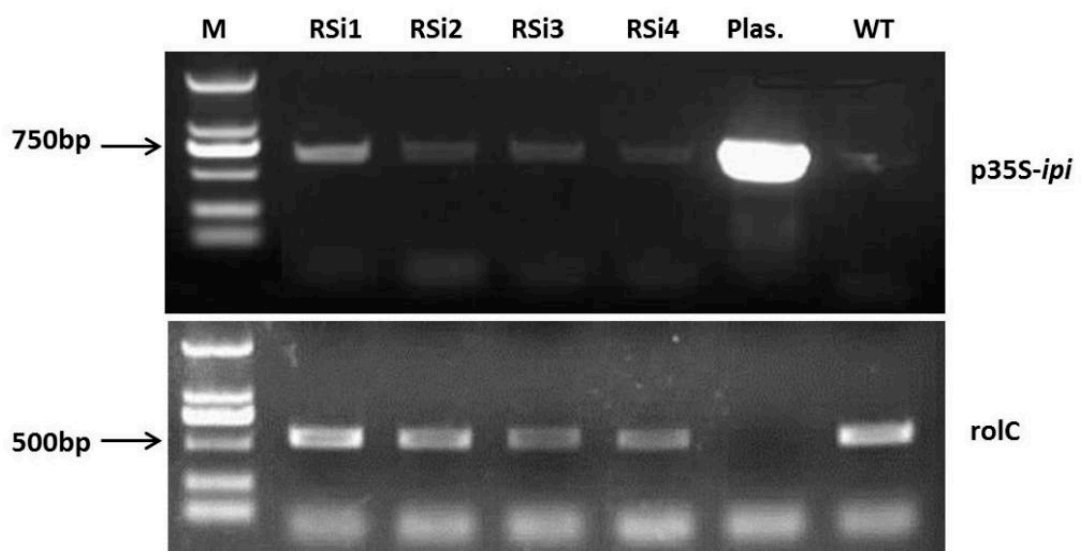

Figure 6. (Aa) under white light of fluorescence microscopy; (Ab) under blue excitation light of fluorescence microscopy; (Ac) superposition of white and blue light of fluorescence microscopy; (B) Representative PCR analyses for the presence of p35S-ipi and rolC genes in transgenic hairy root lines. M: DL-2000 marker (100-2000 bp); RSi: transgenic root lines; WT: the wild type of S. miltiorrhiza roots (negative control); Plas: the vector of pK7GWIWG2D-ipi (positive control plasmid).

\subsection{Phenotype, mRNA Expression, and Terpenoid Metabolites Content of RNAi Hairy Roots}

S. miltiorrhiza hairy roots extended on the edge of leaves (Figure 7Aa), single root was transferred to solid MS medium individually (Figure 7Ab). It was subcultured in shake flasks with 6,7-V liquid medium and then was removed two month later. The WT hairy roots grew denser and brown with lots of branches (Figure 7Ac).

On the other hand, the RNAi roots also emerged on the edge of cutting leaves at the beginning, while it grew very slowly when it was separately subcultured on MS solid medium (Figure 7Ba). Cultured for two months in 6,7-V liquid medium, the texture of hairy roots become very brittle, and it grew few branches (Figure 7Bb). Actually a large number of interference lines have been established, but only a few of them survived, most of the hairy roots died in the process of subculture in shake flasks. For the rest, four lines were randomly selected to be monitored. The mRNA expression levels 
of SmIPI1 in the four lines were significantly lower than that of the WT. In RNAi line RSi3, it was was only $8.4 \%$ of that in the WT roots (Figure $8 \mathrm{~A}$ ), which was the lowest level among the four detected lines. The content of four kinds of terpenoids was detected. The total tanshinone (TT) content in the RNAi line RSi3 was $1.68 \mathrm{mg} / \mathrm{g}$ dry weight, which was significantly lower than $15.55 \mathrm{mg} / \mathrm{g}$ dry weight in the WT control. The TT contents of the RSi1, RSi2, and RSi4 lines could not be detected (Figure 8B, Table 1). These results suggest that TT accumulation was severely affected due to SmIPI1 interference. Dihydrotanshinone I, cryptotanshinone, tanshinone I, and tanshinone IIA are the main diterpene tanshinones of S. miltiorrhiza, which are generated primarily from the MEP metabolic pathway. It can be preliminary deduced from these results that the IPI1 is essential to the plastid MEP pathway of S. miltiorrhiza, and that RNAi of SmIPI1 in the hairy roots would lead to a sharp reduction of metabolites.
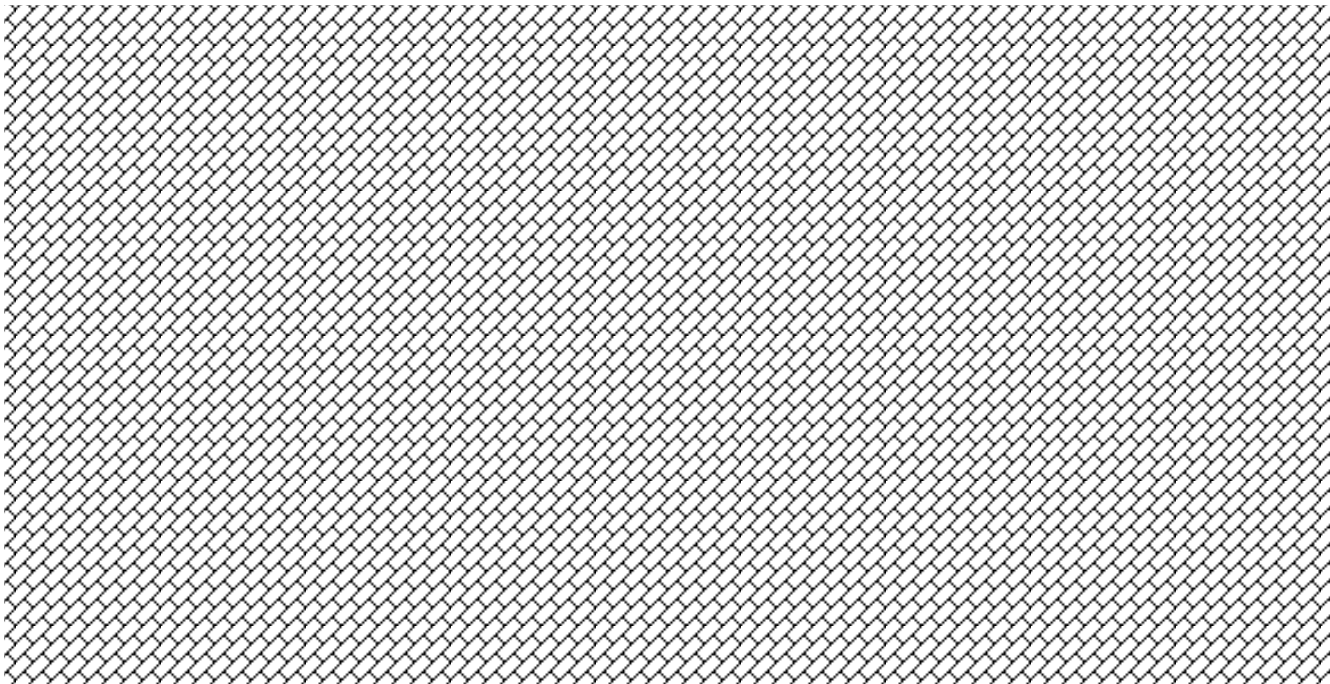

Figure 7. Phenotype comparisons between the RNA interference lines and WT hairy roots. (Aa) WT hairy root emerged on the edge of leaves; (Ab) single hairy root grew many branches when subcultured on MS solid medium; (Ac) WT hairy roots were removed from 6,7-V liquid medium after two month; (Ba) single RNAi hairy root was transferred to MS solid medium; (Bb) SmIPI1 interference hairy roots were removed from shake flasks cultures after two months.

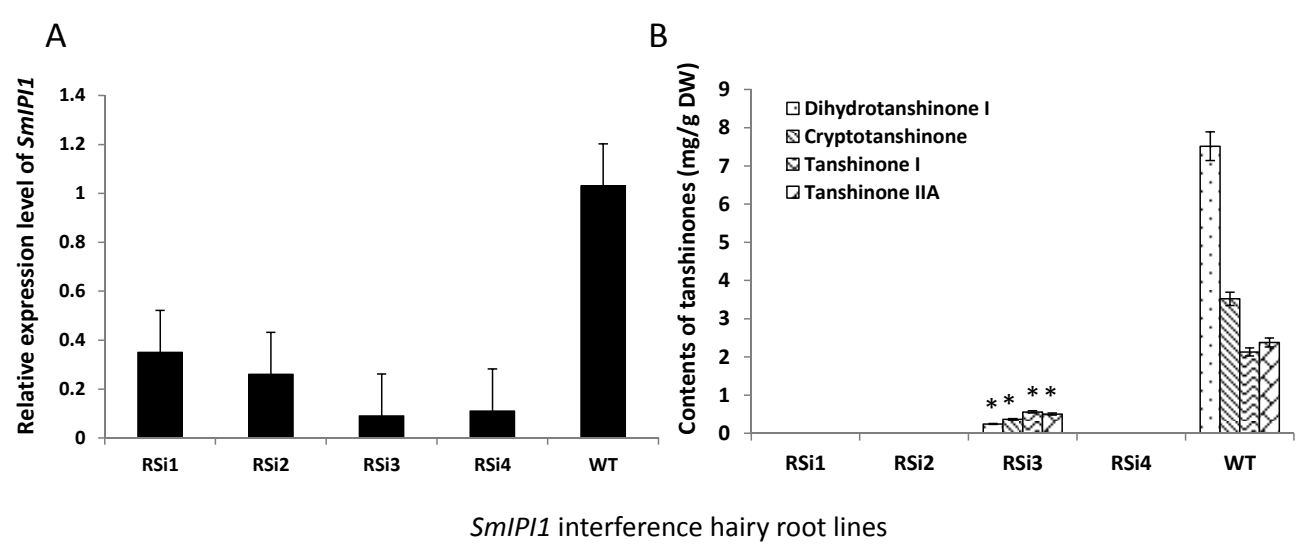

Figure 8. (A) The mRNA expression level SmIPI1 in RNAi hairy root lines and WT; (B) Contents of four kinds of tanshinones between RNA interference lines and WT. The values are means \pm SD of triplicate analyses. ${ }^{*}$, significant difference at $p<0.05$. 
Table 1. The tanshinones contents in S. miltiorrhiza transgenic hairy roots at the end of the culture time.

\begin{tabular}{ccccc}
\hline \multirow{2}{*}{ Lines } & \multicolumn{4}{c}{ Metabolite contents $\mathbf{~} \mathbf{m g} / \mathbf{g}$ DW) } \\
\cline { 2 - 5 } & Dihydrotanshinone I & Cryptotanshinone & Tanshinone I & Tanshinone IIA \\
\hline RSi1 & nd & nd & nd & nd \\
RSi2 & nd & nd & nd & nd \\
RSi3 & $0.24 \pm 0.13 \mathrm{a}$ & $0.37 \pm 0.28 \mathrm{a}$ & $0.56 \pm 0.05 \mathrm{a}$ & $0.51 \pm 0.14 \mathrm{a}$ \\
RSi4 & nd & nd & nd & nd \\
WT & $7.52 \pm 0.97 \mathrm{c}$ & $3.52 \pm 0.27 \mathrm{~b}$ & $2.13 \pm 0.12 \mathrm{~b}$ & $2.38 \pm 0.24 \mathrm{~b}$ \\
\hline
\end{tabular}

The values represent the means of three independent experiments (means $\pm \mathrm{SD}$ ). nd, not detected. a-c, different letters indicate significant differences $(p<0.05)$.

\section{Materials and Methods}

\subsection{Materials}

All biological materials used in this work were derived from S. miltiorrhiza collected from Shangluo, Shanxi Province, China. Roots, stems, and leaves used for tissue expression pattern analysis were collected from 2-year-old plants in June when the pharmacologically active components were rapidly accumulated [20]. Axenic cultures were prepared after the seeds were surface-sterilized in $0.1 \%$ mercuric chloride (Sigma-Aldrich, Shanghai, Trading Co., Ltd., China). Sterile seeds were seeded on Petri dishes containing MS agar medium for germination. Plants were incubated under a 16-h light/8-h dark illumination regime at $25{ }^{\circ} \mathrm{C}$ with $60 \%$ relative humidity. S. miltiorrhiza hairy roots were derived after the seedlings were infected with Agrobacterium rhizogenes (ACCC 10060) harboring $R i$ T-DNA. Stock cultures of hairy roots were maintained at $25{ }^{\circ} \mathrm{C}$ in the dark on solid hormone-free MS medium with $30 \mathrm{~g} / \mathrm{L}$ sucrose. For $\mathrm{Ag}^{+}$eliciter treatment, subcultured hairy roots were transferred to $6,7-\mathrm{V}$ liquid medium and cultivated for 7 days. Three $\mathrm{mM} \mathrm{Ag} \mathrm{Ag}^{+}$elicitor was added to the medium to obtain a final concentration of $30 \mathrm{mM}$. Hairy roots were harvested at $0,12,24,36$, 48 , and 72 h, respectively. Each treatment was repeated three times, the samples were dried with filter paper, and then $0.1 \mathrm{~g}$ of each sample was stored at $-80^{\circ} \mathrm{C}$ for RNA extraction. Hairy roots for RNAi experiments were maintained as shake-flask cultures in 250-mL Erlenmeyer flasks, each containing $100 \mathrm{~mL}$ medium on an orbital shaker set at $110-120 \mathrm{rpm}$ at $25{ }^{\circ} \mathrm{C}$ in the dark. For this study, the synthesis of all primers and DNA sequencing were carried out at the Beijing Sangon Biotechnological (Beijing, China).

\subsection{DNA and RNA Isolation}

Total RNA was extracted from the tissues by Trizol method (Invitrogen, Carlsbad, CA, USA). Genomic DNA of transgenic hairy roots was isolated using the modified cetyltrimethylammonium bromide method [21] for PCR detection.

\subsection{Bioinformatics Analysis}

A BLAST search was conducted to analyze the SmIPI1 cDNA sequence (GenBank accession number: EF635967) on the National Center for Biotechnology Information website. Sequence homology comparison was performed with ClustalW, and the phylogenetic tree was built using the neighbor-joining method in MEGA5.1.

\subsection{Quantitative Reverse Transcription-Polymerase Chain Reaction (qRT-PCR) Analysis of SmIPI1 Expression Level}

First-strand cDNA was synthesized from $2 \mu \mathrm{g}$ of total RNA, which was detected by $1 \%$ agarose gel electrophoresis according to the manufacturer's protocol (Takara, Dalian, Biotechnology, Co., Ltd., China). A pair of primers (actinF: 5'-AGGAACCACCGATCCAGACA-3', actinR: $5^{\prime}$-GGTGCCCTGAGGTCCTGTT-3') was designed to amplify the housekeeping gene $(\beta$-actin) as 
an internal control, and then the expression level of SmIPI1 in different tissues was detected with semi-quantitative RT-PCR using another pair of primers (SmI1F: 5'-CGTCCTTGACCAGCATC-3', SmI1R: 5'-GGCGTTGGGTTTGGGAT-3'). All experiments (including the RNA extraction) were repeated three times.

\subsection{Reconstruction of the Prokaryotic Expression Vector for Functional Identification of Smipi1 through Color Complementation Analysis}

The pTrc-AtIPI and pAC-LYC vectors were kindly donated by Francis X. Cunningham Jr. (Department of Cell Biology and Molecular Genetics, University of Maryland, College Park, Baltimore, MD, USA). The pTrc-AtIPI vector was the combination of IPI gene from Arabidopsis thaliana (AtIPI), pTrcHisB and pBlueScript SK-. The pTrcHisB vector was digested by restriction enzyme KpnI and EcoRI, and then the coding sequence of AtIPI inserted, Subsquently, the 6x His tag was removed, as well as the fragment located between EcoRV and EcoRI. The fragments with an AtIPI gene and Trc promoter were finally digested using EcoRV and EcoRI and inserted into the pBlueScript SKplasmid. The pTrc-AtIPI vector, as an expression vector, retains an Amp resistance gene. On the other hand, the pAC-LYC prokaryotic expression vector carries three key enzyme genes, phytoene synthase (crtB), geranylgeranyl pyrophosphate synthase (crtE), and phytoene desaturase (crtI), which encode proteins involved in the $\beta$-carotene biosynthetic pathway [22] E. coli cells containing pAC-LYC vectors can survive in LB medium with $C h l$ and produce small amounts of lycopene, resulting in light pink colonies. For functional analysis of SmIPI1, a specific primer pair containing restriction endonuclease sites of NotI and BglII was designed to substitute AtIPI in the pTrc-AtIPI plasmid as follows: trcIPIF 5'-CATAGATCTATGTCGTCCTTGACCAGCATCCCGT- ${ }^{\prime}{ }^{\prime}, \operatorname{trcIPIR} 5^{\prime}$-ATAGCGGC CTAAGTC-3'. The primers were used for PCR amplification with SmIPI1 cDNA as a template. The amplified products were purified and introduced into E. coli $\mathrm{DH} 5 \alpha$ competent cells, and then selected on Amp (50 mg/L), followed by sequencing analysis. The positive clones with accurate sequencing results were expanded and the plasmid was extracted. pTrc-AtIPI was double-digested with NotI and BglII restriction endonucleases and the large fragments ligating with SmIPI1 were recycled and also doubled-digested with the same endonucleases. The ligation product was named pTrc-SmIPI1.The correct pTrc-SmIPI1 plasmid was extracted after selection on antibiotic plates and then sequenced. The recombinant plasmid pTrc-SmIPI1 was transformed into XL1-blue competent cells containing the pAC-LYC-engineered bacteria, and streaked onto LB solid medium with Amp $(100 \mathrm{mg} / \mathrm{L})$ and $\mathrm{Chl}(30 \mathrm{mg} / \mathrm{L})$. Plasmids carrying the double-engineered bacteria that could produce lycopene were obtained. At the same time, the empty vector pTrc and engineered pAC-LYC plasmid were introduced into XL1-blue competent cells to obtain the control strain. Plates were incubated at $37^{\circ} \mathrm{C}$ for $24 \mathrm{~h}$ and then at $28^{\circ} \mathrm{C}$ for 3 days in the dark to allow time for sufficient lycopene accumulation to be able to observe obvious color differences.

\subsection{Construction of Interfering Plasmids}

Based on the protocol of Gateway Clone Technology (Invitrogen), the attB sequence primers RiBF-ipi (5'-GCACAAGTTTGTACAAAAAAGCAGGCTGGACGCCGTTCAGAGGCGCCTC-3') and RiBR-ipi (5'-GCACCACTTTGTACAAGAAAGCTGGGTTCCTCTGAGCAGCATTCCTC AC-3') were used to amplify the 310-bp fragment of SmIPI1 (ipi, located from coding sequence positions $240-550 \mathrm{bp}$ ) that carries an adjoining attB sequence. Using the right-sequencing attB-ipi purified product as a template, the BP clonase II enzyme mix (Invitrogen) was used to construct the entry vector pDONR221-ipi. The BP reaction plasmid was used to react with the interfering vector pK7GWIWG2D (Figure 9), adopting the LR clonase II enzyme mix according to the manufacturer's instructions. Products were transformed into $\mathrm{DH} 5 \alpha$ cells for multiplication with the heat shock method, and then resistance-screened on LB medium containing $50 \mu \mathrm{g} / \mathrm{mL}$ Spe. The Kan-resistant gene was used as the screening gene and the enhanced green fluorescent protein (Egfp) coding gene 
was used as the reporter gene owing to its visibility and nondestructive property. Wild-type (WT) hairy roots were regarded as the common control for RNAi lines.

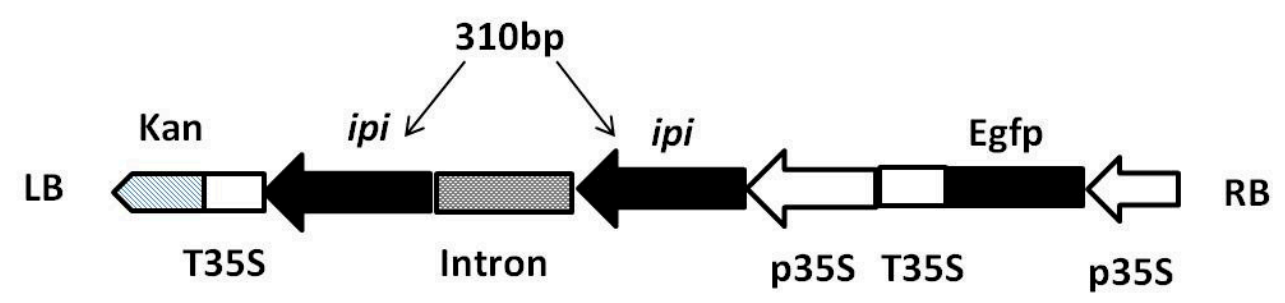

Figure 9. Construction of the pK7GWIWG2D-ipi RNA interference vector. p35S, a promoter region from the cauliflower mosaic virus (CaMV) 35S gene; T35S, a terminator fragment from the CaMV $35 \mathrm{~S}$ gene; Egfp, enhanced green fluorescent protein; ipi, 310-bp fragment of SmIPI1 used for interference plasmid construction; LB, left border; RB, right border.

\subsection{Agrobacterium rhizogenes ACCC 10060 Mediated Gene Transformation into S. miltiorrhiza Hairy Roots}

The freeze-thaw method was adopted to transform the plasmid into A. rhizogenes ACCC 10060 competent cells. The induction and culturing methods for S. miltiorrhiza hairy roots were described previously [23]. Fast-growing antibiotic-resistant hairy roots of $2.0-3.0 \mathrm{~cm}$ long were chosen for cutting of a single root and numbered. RNAi hairy roots were screened and cultured on a solid medium of MS containing $400 \mathrm{mg} / \mathrm{L}$ Cef and $50 \mathrm{mg} / \mathrm{L}$ Kan.

\subsection{Green Fluorescent Protein Detection and PCR Verification}

The hairy roots growing from the antibiotic plates were placed under an inverted fluorescence microscope to observe green fluorescence excitation. Furthermore, specific primers, 35S-Ripi1 (5'-ATCTAACAGAACTCGCCGTGAA-3') and 35S-Ripi2 (5'-ACTGAAAGCTCCGTGCAACA-3'), were designed to detect the insertion of the external ipi fragment linked to the p35S sequence in the interference lines. The primers RolCF (5'-CTCCTGACATCAAACTCGTC- $\left.3^{\prime}\right)$ and RolCR (5'-TGCTTCGAGTTATGGGTACA-3') were used to detect the rolC gene of A. rhizogenes, and the positive roots were chosen to be amplified and cultured.

\subsection{Measurement of Tanshinones Content}

The hairy roots subcultured for 60 days in 6,7-V liquid medium were harvested for tanshinones measurement. The extraction and measuring methods of deterpene components followed those described by Dai et al. [23]. The data are expressed as the mean \pm SD value of three independent experiments. Comparison of multiple groups was conducted using one-way analysis of variance (ANOVA), and $p<0.05$ was considered statistically significant.

\section{Discussion}

IPI acts as the general switch of the terpene downstream metabolic pathway, which directly influences the trend of terpenoid metabolic flux [24]. Many studies have demonstrated a significant increase in the content of isoprenes after the transfer of an exogenous IPI gene into E. coli. Therefore, IPI has become an effective target for the metabolic engineering of isoprenes in E. coli [25]. However, few studies have examined the IPI enzyme in plants. Therefore, we conducted a series of experiments in order to reveal the function of IPI in terpenoid metabolism from several aspects.

In the last decade, $\mathrm{Ag}^{+}$has been reported to be an effective elicitor that could significantly improve the accumulation of tanshinones [26,27]. In our study, the relative expression level of SmIPI1 showed a parabolic trend of variation over time after induction with $\mathrm{Ag}^{+}$, indicating that the tanshinones accumulation of S. miltiorrhiza may be closely related to the expression of SmIPI1. 
The recombinant vector pTrc-SmIPI1 was verified to facilitate increased lycopene synthesis via the action of a strong promoter, trc, in E. coli. Lycopene is a linear carotenoid responsible for the red color in some fruits and vegetables such as tomatoes and watermelon, and contains seven IPP and one DMAPP molecules in its structure. The color of a carotenoid is principally a consequence of the number of conjugated double bonds. As the number of double bonds increases, the carotenoid light absorbance shifts further to the red region of the spectrum [28]. On a dual resistance medium, the bacteria clones containing the double plasmids pAC-LYC and pTrc-SmIPI1 presented a deeper red color than those containing PAC-LYC and PTrc, which indicated that more lycopene had accumulated. These results confirmed that SmIPI1 promotes metabolic flux to the biosynthesis pathway of lycopene. According to the recently published genome data of S. miltiorrhiza [29], there are two IPI cDNAs in S. miltiorrhiza. The same method was applied to SmIPI2 (another homologous SmIPI gene) to confirm the action of both genes. Unfortunately, the color of engineered E. coli harboring the pTrc-SmIPI2 plasmid did not show any obvious changes. In Arabidopsis and tobacco, it has been demonstrated that the two IPI genes (IPI1 and IPI2) were targeted to different organelles and played distinct roles in isoprenoid metabolism [30,31]. The SmIPI2 gene may also have different functions in S. miltiorrhiza, which require further studies for confirmation.

After genetic transformation of $S$. miltiorrhiza hairy roots, the phenotype of transgenic root lines, changes in mRNA levels of key enzymes, and the metabolic characteristics of RNAi SmIPI1 lines were analyzed. The gene expression level of SmIPI1 in the RNAi lines was significantly lower than that of the WT. The fragment at 240-550 bp (corresponding to 80-183 aa) in the SmIPI1 sequence that we chose for constructing the interference vector is a relatively conserved region with respect to the IPI genes of other plants. Therefore, the results obtained in this study likely reflect interference of both IPI genes. Interference of SmIPI1 in the hairy roots resulted in an obvious abnormal phenotype characterized by withering and deformation. It can be inferred that interference of IPI gene expression in plants will be a fatal blow to the isoprenoid metabolic pathway, which can seriously affect the growth of plants and interrupt terpenoid metabolism. Maryer et al. [16] discovered cell death after knocking out the IPI gene (single-copy gene) in Saccharomyces cerevisiae. This result illustrated that although the IPI protein itself is not required for cell division or spore germination, the cells are unable to survive once the internal pool of isoprenoid metabolites is depleted. Our research is similar to that of the previous study. It contributes novel information to verify that SmIPI1 plays an important role in the MEP metabolic pathway. Our research will provide a basis for further study on the function of IPI.

\section{Conclusions}

In this study, lycopene engineering bacteria has been used to discover the function of SmIPI1 in E. coli. In addition, based on the genetic transformation of S. miltiorrhiza hairy roots, phenotype of transgenic hairy roots, changes of mRNA level of SmIPI1 and metabolic characteristics of SmIPI1 RNAi hairy roots were analyzed. It is now clearly established that the IPI is crucial in terpenoid synthesis. The study on the IPI function in plants will further figure out the terpenoid metabolic pathway clearly.

Acknowledgments: This work was supported by the National Natural Science Foundation of China (no. 81303166).

Author Contributions: Lu-Qi Huang designed research; Xia-Nan Zhang, Hong-Yu Guan, Zhu-Bo Dai, Juan Guo, Ye Shen, Guang-Hong Cui, Wei Gao performed research and analyzed the data; Xia-Nan Zhang, Hong-Yu wrote the paper. All authors read and approved the final manuscript.

Conflicts of Interest: The authors declare no conflict of interest.

\section{References}

1. Xu, Z. Modernization: One step at a time. Nature 2011, 480, S90-S92. [CrossRef] [PubMed] 
2. Ren, D.; Du, G.; Zhang, J. Protective effect of total salvianolic acid against cerebral ischemia reperfusion injury. Chin. Pharmacol. Bull. 2002, 18, 219-221.

3. Tian, P. Convergence: Where West meets East. Nature 2011, 480, S84-S86. [CrossRef] [PubMed]

4. Koyama, T.; Ogura, K. Comprehensive Natural Product Chemistry; Cane, D.E., Ed.; Elsevier Science Ltd.: Oxford, UK, 1999; pp. 69-96.

5. Kaneda, K.; Kuzuyama, T.; Takagi, M.; Hayakawa, Y.; Seto, H. An unusual isopentenyl diphosphate isomerase found in the mevalonate pathway gene cluster from Streptomyces sp. strain CL190. Proc. Natl. Acad. Sci. USA 2001, 98, 932-937. [CrossRef] [PubMed]

6. Takagi, M.; Kaneda, K.; Shimizu, T.; Hayakawa, Y.; Seto, H.; Kuzuyama, T. Bacillus subtilis ypgA gene is fni, a nonessential gene encoding type 2 isopentenyl diphosphate isomerase. Biosci. Biotechnol. Biochem. 2004, 68, 132-137. [CrossRef] [PubMed]

7. Barkley, S.J.; Desai, S.B.; Poulter, C.D. Type II isopentenyl diphosphate isomerase from Synechocystis sp. strain PCC 6803. J. Bacteriol. 2004, 186, 8156-8158. [CrossRef] [PubMed]

8. Okada, K.; Kasahara, H.; Yamaguchi, S.; Kawaide, H.; Kamiya, Y.; Nojiri, H.; Yamane, H. Genetic evidence for the role of isopentenyl diphosphate isomerases in the mevalonate pathway and plant development in Arabidopsis. Plant Cell Physiol. 2008, 49, 604-616. [CrossRef] [PubMed]

9. Anderson, M.S.; Muehlbacher, M.; Street, I.P.; Proffitt, J.; Poulter, C.D. Isopentenyl diphosphate: Dimethylallyl diphosphate isomerase. An improved purification of the enzyme and isolation of the gene from Saccharomyces cerevisiae. J. Biol. Chem. 1989, 264, 19169-19175. [PubMed]

10. Hahn, F.M.; Hurlburt, A.P.; Poulter, C.D. Escherichia coli open reading frame 696 is idi, a nonessential gene encoding Isopentenyl Diphosphate Isomerase. J. Bacteriol. 1999, 181, 4499-4504. [PubMed]

11. Blanc, V.M.; Pichersky, E. Nucleotide sequence of a Clarkia breweri cDNA clone of Ipi1, a gene encoding isopentenyl pyrophosphate isomerase. Plant Physiol. 1995, 108, 855-856. [CrossRef] [PubMed]

12. Sun, Z.; Cunningham, F.X., Jr.; Gantt, E. Differential expression of two isopentenyl pyrophosphate isomerases and enhanced carotenoid accumulation in a unicellular chlorophyte. Proc. Natl. Acad. Sci. USA 1998, 95, 11482-11488. [CrossRef] [PubMed]

13. Yoon, S.H.; Lee, Y.M.; Kim, J.E.; Lee, S.H.; Lee, J.H.; Kim, J.Y.; Jung, K.H.; Shin, Y.C.; Keasling, J.D.; Kim, S.W. Enhanced lycopene production in Escherichia coli engineered to synthesize isopentenyl diphosphate and dimethylallyl diphosphate from mevalonate. Biotechnol. Bioeng. 2006, 94, 1025-1032. [CrossRef] [PubMed]

14. Kajiwara, S.; Fraser, P.D.; Kondo, K. Expression of an exogenous isopentenyl diphosphate isomerase gene enhances isoprenoid biosynthesis in Escherichia coli. Biochem. J. 1997, 324, 421-426. [CrossRef] [PubMed]

15. Sun, J.; Zhang, Y.Y.; Liu, H.; Zou, Z.; Zhang, C.J.; Zhang, X.H.; Li, H.X.; Ye, Z.B. A novel cytoplasmic isopentenyl diphosphate isomerase gene from tomato (Solanum lycopersicum): Cloning, expression, and color complementation. Plant Mol. Biol. Rep. 2010, 28, 473-480. [CrossRef]

16. Mayer, M.P.; Hahn, F.M.; Stillman, D.J.; Poulter, C.D. Disruption and mapping of IDI1, the gene for isopentenyl diphosphate isomerase in Saccharomyces cerevisiae. Yeast 1992, 8, 743-748. [CrossRef] [PubMed]

17. Cui, G.H.; Huang, L.Q.; Tang, X.J.; Zhao, J.X. Candidate genes involved in tanshinone biosynthesis in hairy roots of Salvia miltiorrhiza revealed by cDNA microarray. Mol. Biol. Rep. 2011, 38, 2471-2478. [CrossRef] [PubMed]

18. Zhang, S.; Liu, Y.; Shen, S.; Liang, Z.; Yang, D. Effects of elicitors on accumulation of phenolic acids and tanshinones in Salvia miltiorrhiza hairy root. China J. Chin. Mater. Med. 2011, 36, 1269-1274.

19. Cunningham, F.X.; Pogson, B.; Sun, Z.R.; McDonald, K.A.; DellaPenna, D.; Gantt, E. Functional analysis of the beta and epsilon lycopene cyclase enzymes of Arabidopsis reveals a mechanism for control of cyclic carotenoid formation. Plant Cell. 1996, 8, 1613-1626. [CrossRef] [PubMed]

20. Xu, C.; Shu, Z.; Wang, Y.; Miao, F.; Zhou, L. The accumulation rule of the main medicinal components in different organs of Salvia miltiorrhiza Bunge. and Salvia miltiorrhiza Bunge. f. alba. Lishizhen Med. Mater. Med. Res. 2010, 21, 2129-2132.

21. Del, S.G.; Manfioletti, G.; Schneider, C. The CTAB-DNA precipitation method: A common miniscale preparation of template DNA from phagemids, phages or plasmids suitable for sequencing. Biotechniques 1989, 7, 514-519.

22. Cunningham, F.X.; Sun, Z.R.; Chamovitz, D.; Hirschberg, J.; Gantt, E. Molecular structure and enzymatic function of lycopene cyclase from the Cyanobacterium Synechococcus sp strain PCC7942. Plant Cell 1994, 6, 1107-1121. [CrossRef] [PubMed] 
23. Dai, Z.B.; Cui, G.H.; Zhou, S.F.; Zhang, X.N.; Huang, L. Cloning and characterization of a novel 3-hydroxy-3-methylglutaryl coenzyme A reductase gene from Salvia miltiorrhiza involved in diterpenoid tanshinone accumulation. J. Plant Physiol. 2011, 168, 148-157. [CrossRef] [PubMed]

24. Pan, X.C.; Chen, M.; Liu, Y.; Liao, Z.H. Cloning of Deoxyoxylulose-5-phosphate Synthase Gene from Tomato and Its Color Complemention in E. coli. Acta Hortic. Sin. 2007, 34, 371-376.

25. Jomaa, H.; Wiesner, J.; Sanderbrand, S. Inhibitors of the nonmevalonate pathway of isoprenoid biosynthesis as antimalarial drugs. Science 1999, 285, 1573-1576. [CrossRef] [PubMed]

26. Zhang, X.N.; Guo, J.; Shen, Y.; Huang, L.Q. Cloning and expression analysis of a new 3-hydroxy-3-methylglutaryl coenzyme A reductase gene from Salvia miltiorrhiza (SmHMGR3). China J. Chin. Mater. Med. 2012, 37, 2378-2382.

27. Jiang, L.Z.; Li, G.Z.; Jian, Y.W. Effects of biotic and abiotic elicitors on cell growth and tanshinone accumulation in Salvia miltiorrhiza cell cultures. Appl. Microbiol. Biotechnol. 2010, 87, 137-144.

28. Garcia-Asua, G.; Lang, H.P.; Cogdell, R.J. Carotenoid diversity: A modular role for the phytoene desaturase step. Trends Plant Sci. 1998, 3, 445-449. [CrossRef]

29. Ma, Y.; Yuan, L.; Wu, B.; Li, X.; Chen, S.; Lu, S. Genome-wide identification and characterization of novel genes involved in terpenoid biosynthesis in Salvia miltiorrhiza. J. Exp. Bot. 2012, 63, 2809-2823. [CrossRef] [PubMed]

30. Nakamura, A.; Shimada, H.; Masuda, T.; Ohta, H.; Takamiya, K. Two distinct isopentenyl diphosphate isomerases in cytosol and plastid are differentially induced by environmental stresses in tobacco. FEBS Lett. 2001, 506, 61-64. [CrossRef]

31. Phillips, M.A.; D'Auria, J.C.; Gershenzon, J.; Pichersky, E. The Arabidopsis thaliana type I Isopentenyl Diphosphate Isomerases are targeted to multiple subcellular compartments and have overlapping functions in isoprenoid biosynthesis. Plant Cell 2008, 20, 677-696. [CrossRef] [PubMed]

Sample Availability: Samples of the plasmids pAC-LYC, pTrc-AtIPI and pTrc-SmIPI1 are available from the authors.

(C) 2015 by the authors; licensee MDPI, Basel, Switzerland. This article is an open access article distributed under the terms and conditions of the Creative Commons by Attribution (CC-BY) license (http://creativecommons.org/licenses/by/4.0/). 